\title{
USG guided FNAC of ovarian mass lesions: A cyto-histopathological correlation, with emphasis on its role in pre-operative management guidelines
}

\author{
Sailesh Ray ${ }^{1}$, Mimi Gangopadhyay², Arghya Bandyopadhyay ${ }^{4}$, Kaushik Majumdar ${ }^{3}$, Nilanjana Chaudhury ${ }^{1}$ \\ 'Department of Gynaecology and Obstetrics, N. B. Medical College, Darjeeling, India \\ ${ }^{2}$ Department of Pathology, N. B. Medical College, Darjeeling, India \\ ${ }^{3}$ Department of Pathology, G. B. Pant Hospital, New Delhi, India \\ ${ }^{4}$ Department of Pathology, Burdwan Medical College, Burdwan, India
}

\section{Abstract}

Objective: Ultrasonography (USG)-guided fine-needle aspiration cytology (FNAC) of ovarian masses is an efficient diagnostic modality for accurately diagnosing ovarian tumours prior to surgery. The main aim of this study was to assess the sensitivity, specificity and accuracy of FNAC in diagnosing ovarian masses.

Material and Methods: Eighty-three patients with ovarian masses were recruited and correlation of USG-guided FNAC was made with histopathology in all but 6 cases, where surgery was not indicated.

Results: Cytological diagnosis was obtained in all 83 ovarian lesions: 56 cases were benign, 6 possibly benign, 3 suspicious of malignancy and 18 cases as malignant. Out of 77 cases where histology was available, the 12 non-neoplastic cysts were endometriotic cysts and follicular cysts. The majority of neoplastic lesions were surface epithelial tumours. Out of 12 non-neoplastic cysts and 43 benign tumours, all but two were diagnosed as benign or possibly benign on cytology; of the 22 histologically malignant or borderline tumours, 18 were malignant or suspicious of malignancy on cytology, while four were false negative (three of these were borderline tumours). Thus, the sensitivity of cytological diagnosis was $83 \%$, specificity was $97 \%$ and accuracy was $93 \%$.

Conclusion: USG-guided FNAC seems to be a relatively safe, simple, fast and cost-effective procedure where most ovarian malignancies either present late in their course or no screening method is available. In addition, cyto-radiological correlation through this procedure may be useful in deciding management guidelines prior to any surgical intervention.

(J Turk Ger Gynecol Assoc 2014; 15: 6-12)

Key words: Ultrasound, fine-needle aspiration cytology, ovarian mass, image-guided, cytology

Received: 31 October, 2013

Accepted: 19 December, 2013

\section{Introduction}

With the increased use of imaging techniques, the detection of asymptomatic ovarian masses has risen considerably. The nature of ovarian lesions less than 5-6 $\mathrm{cm}$ may be difficult to determine and cysts in premenopausal women may be functional. However, an ovarian mass in perimenopausal and menopausal women is a matter of concern, owing to the increased risk of malignancy in this age group (1). The incidence of malignant ovarian masses has steadily increased over the past two decades and is one of the leading causes of cancer-related deaths globally. The majority $(>90 \%)$ of ovarian masses are benign but almost two-thirds of malignant ovarian tumours present at an advanced stage (stage III or IV) (2). Clinicians are aware of the difficulty in differentiating a unilocular cystadenoma and a follicular or corpus luteum cyst in the clinical setting (3). Unfortunately, no single test or combination of tests has been shown to accurately predict ovarian histologic findings. Limitations of our current techniques might be the cause of many unnecessary surgical interventions which could have been otherwise avoided (1). Fine-needle aspiration cytology (FNAC) of these ovarian cysts may offer the potential to decrease the need for surgical procedures in these women. Historically, gynaecologists have been hesitant to aspirate ovarian cysts in view of the possibility of seeding an early stage ovarian cancer. The magnitude of risk of such a procedure is unknown and not substantiated by convincing evidence. It is rather overestimated and has not been pathologically confirmed (4). Some researchers believe that ovarian cysts can be safely aspirated for diagnos- 
tic purposes, and that FNAC should be used in conditions like suspected benign ovarian cysts and recurrent and metastatic tumours, when the patient's condition is unsuitable for surgery $(1,5)$. Among the available imaging modalities, ultrasonography (USG) is economical, rapid and widely available, not only providing substantial information regarding the nature of the mass but also guiding the fine-needle aspiration (FNA) with adequate precision (6). Previous studies have attempted to estimate the efficacy of image-guided FNAC in the accurate preoperative diagnosis of ovarian lesions $(1,5-8)$. The main aim of this study was to assess the sensitivity, specificity and diagnostic accuracy of USG-guided FNAC in the distinction of neoplastic and non-neoplastic ovarian masses, considering histopathology as the gold standard.

\section{Material and Methods}

Patients presenting with ovarian masses diagnosed clinically (abdominal and per vaginal examination) and/or by USG during the period from September 2008 to March 2011 were included in this study. FNAC under USG guidance was performed through the abdominal or trans-vaginal route for incidentally detected localised mass lesions, benign cystic lesions or advanced malignant neoplasms. Informed consent was obtained from the patients, mentioning that the procedure was carried out for diagnostic purposes and to decide upon further management. Ethical committee approval was obtained before the study was commenced. The ovarian lesions were aspirated using a 20 $\mathrm{ml}$ syringe fitted with a 22-gauge long needle. Air-dried smears were prepared and stained with May-Grünwald-Giemsa (MGG) and wet-fixed smears were subjected to Papanicolaou and/or H\&E (Haematoxylin \& Eosin) stain. In cases where cyst fluid was aspirated, it was subjected to cytocentrifugation and the sediment was stained by similar methods. Patients suspected of harbouring malignant lesions based on menopausal status and suggestive USG findings were subjected to serum CA-125 estimation. In all but 6 cases, surgery was performed within 10 days of FNAC. The resected ovarian mass lesions were routinely processed and the tumours were histologically classified as per the guidelines established by the World Health Organisation (WHO) classification. Based on clinico-radiological correlation, the cytological findings were categorised as benign, possibly benign, suspicious of malignancy and malignant. Based on the cytological diagnosis, the cases were grouped into the following categories: 1 for malignant, 2 for 'suspicious of malignancy' and 3 for 'possibly benign' and benign lesions. Similarly, for histological diagnosis, 1 was designated for malignant SOLs, 2 for the borderline malignant category and 3 for benign lesions. Unilocular cysts less than $5 \mathrm{~cm}$ where only straw-coloured fluid was aspirated were classified as possibly benign. Specimens with cells with a mild increase in the nuclear/cytoplasmic ratio or mild nuclear atypia was assigned as suspicious of malignancy. In addition, background showing tumour diathesis and debris were also considered as features suspicious of malignancy. Cytological features indicating malignancy were high cellularity with cells in three-dimensional clusters and dissociation, nuclear pleomorphism, increase in nuclear-cytoplasmic ratio, prominent nucleoli, presence of mitotic figures and tumour diathesis. Specific categorisation of the lesions was also possible on cytology in cases with certain characteristic findings on smears.

Descriptive statistics were used to determine correlation between cytological and histological findings. Sensitivity and specificity for the cytological diagnoses were calculated using the histological confirmation as the gold standard. Analyses were performed using the SPSS software, version 16.0 (SPSS Inc., Chicago, IL, USA).

\section{Results}

During the 30 month period, 83 cases of ovarian mass lesions were evaluated on cytological smears; of these cases, histopathology was not available in 6 patients. The mean age of the women was 39.8 years (age range 15-70 years). The most common presenting feature was abdominal mass (68.83\%) followed by lower abdominal pain (41.6\%), menstrual disturbances (28.57\%) and weight loss (11.68\%). USG helped in the assessment of the type (cystic, solid, unilocular or multilocular), size, location and extent of the lesion. In case of malignant neoplasms diagnosed during laparotomy, all were beyond stage IIC, except in one patient who had stage IA disease. On image-guided aspiration cytology, 56 cases were diagnosed as benign, 6 as possibly benign, 3 as suspicious of malignancy and 18 cases as malignant.

Histological confirmation was available in 77 cases. Of the 12 non-neoplastic cysts and 43 benign neoplasms on histology, all but two were benign or possibly benign on cytology; of the 22 histologically malignant or borderline tumours, 18 were malignant or suspicious of malignancy on cytology while four were false negatives (three of these were borderline tumours) (Table 1, 2).

All but one of the non-neoplastic cystic lesions were diagnosed accurately by FNAC, which included 2 cases reported as possibly benign and 5 more cases where histopathology was not performed since the cyst dimension was less than or equal to $5 \mathrm{~cm}$. These latter 5 cases of follicular cyst were diagnosed as 'benign cystic lesion' on cytology. One endometriotic cyst was erroneously diagnosed as a serous carcinoma, while the remaining 6 endometriotic cysts were diagnosed accurately. Another case where histology was not available was a serous cystadenocarcinoma with metastasis and peritoneal nodules. For all 83 cases, the sensitivity and specificity of FNAC considering final (histological) diagnosis as the gold standard (Table 3) were $83 \%$ and $97 \%$, respectively, with a diagnostic accuracy of $93 \%$. Chi square test was performed to correlate between cytological and final (histological) diagnosis, and was highly significant $(\mathrm{p}<0.001)$. The measure of agreement between the diagnoses, as obtained by 'Kappa' value, was 0.725 , indicating substantial agreement between the cytological and histological diagnoses. 
Table 1. Comparative analysis of confirmed benign ovarian lesions (on histology) and their corresponding cytological diagnoses

\begin{tabular}{|c|c|c|c|}
\hline $\begin{array}{l}\text { Histopathological Diagnoses } \\
\text { Non-neoplastic lesions }\end{array}$ & \multicolumn{3}{|c|}{ Cytopathological Diagnoses } \\
\hline Follicular cyst $(n=4)$ & $2+5^{*}$ & 2 & \\
\hline Endometriotic cyst $(n=7)$ & 6 & & Serous carcinoma - 1 \\
\hline \multicolumn{4}{|l|}{ Benign neoplasms } \\
\hline Serous cystadenoma $(n=17)$ & 17 & & \\
\hline Mucinous cystadenoma $(n=14)$ & 14 & & \\
\hline Benign cystic teratoma $(n=9)$ & 9 & & \\
\hline Fibroma $(n=2)$ & 2 & & \\
\hline Total $(\mathrm{n}=55)$ & $51+5^{*}$ & 2 & 2 \\
\hline
\end{tabular}

Table 2. Comparative analysis of confirmed benign ovarian lesions (on histology) and their corresponding cytological diagnoses

\begin{tabular}{|c|c|c|c|}
\hline \multirow[b]{2}{*}{ Histopathological Diagnoses } & \multicolumn{3}{|c|}{ Cytopathological Diagnoses } \\
\hline & Malignant & Suspicious of malignancy & False negative \\
\hline Serous cystadenocarcinoma $(n=7)$ & $7+1 *$ & & \\
\hline Mucinous cystadenocarcinoma $(n=4)$ & 3 & & Serous cystadenoma - 1 \\
\hline Serous borderline tumour $(\mathrm{n}=4)$ & 1 & 2 & Serous cystadenoma - 1 \\
\hline \multirow{2}{*}{ Mucinous borderline tumour $(\mathrm{n}=3)$} & \multirow{2}{*}{0} & \multirow{2}{*}{1} & Serous cystadenoma - 1 \\
\hline & & & Mucinous cystadenoma - 1 \\
\hline Dysgerminoma $(\mathrm{n}=1)$ & 1 & & \\
\hline Yolk sac tumour $(n=1)$ & 1 & & \\
\hline Granulosa cell tumour $(\mathrm{n}=1)$ & 1 & & \\
\hline Squamous cell carcinoma $(\mathrm{n}=1)$ & 1 & & \\
\hline Total $(\mathrm{n}=22)$ & $15+1^{*}$ & 3 & 4 \\
\hline
\end{tabular}

Table 3. Correlation of ovarian image guided FNAC and histological (confirmed) diagnoses $(n=83)$

\begin{tabular}{|l|c|c|c|}
\hline \multirow{2}{*}{ Histological Diagnosis } & \multicolumn{3}{|c|}{ FNAC Diagnosis } \\
\cline { 2 - 4 } & Malignant & 2 & 21 \\
\hline Malignant & $19 *$ & $58^{\#}$ & 62 \\
\hline Benign & 4 & 60 & 83 \\
\hline Total & 23 & \\
\hline $\begin{array}{l}\text { FNAC: Fine-needle aspiration cytology } \\
\text { *Includes one advanced case without histology } \\
\text { \#Includes 5 cases <5 cm, which were not biopsied } \\
\text { - Confirmed diagnosis either on histology or clinico-radiological analysis, where histology was not available } \\
\text { - Malignant in FNAC also includes lesions suspicious of malignancy; benign also includes 'possibly benign' lesions }\end{array}$ \\
\hline
\end{tabular}

When characteristic cytomorphological findings were appreciated, the specific diagnosis (instead of merely 'benign', 'possibly benign' or 'malignant') could be offered on cytology. The diagnosis of endometriotic cysts was offered on cytology when sheets of epithelial cells and spindle (stromal) cells were seen against a haemorrhagic background containing haemosiderin- laden macrophages (Figure 1). Out of 77 cases where histology was available, the majority of cases were surface epithelial tumours of serous and mucinous variety (Table 1,2). Serous cystadenomas on FNA yielded straw-coloured fluid. Smears prepared from the centrifuged deposit showed few papillary fragments with bland nuclei and cyst macrophages. Benign 


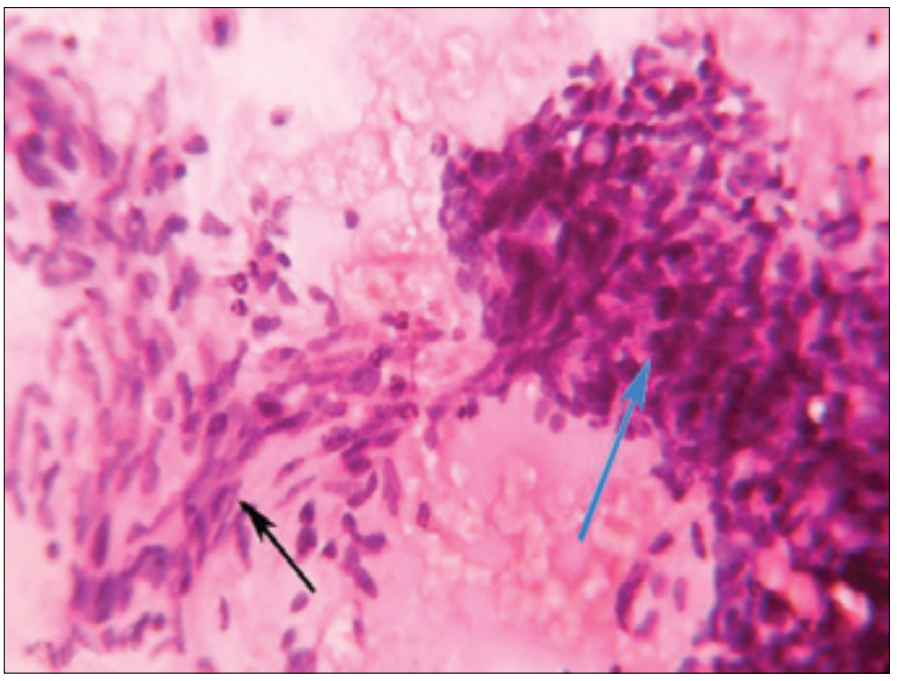

Figure 1. Stromal component (black arrow) \& epithelial component (blue arrow), (Endometriotic cyst), H\&E, $40 \mathrm{X}$

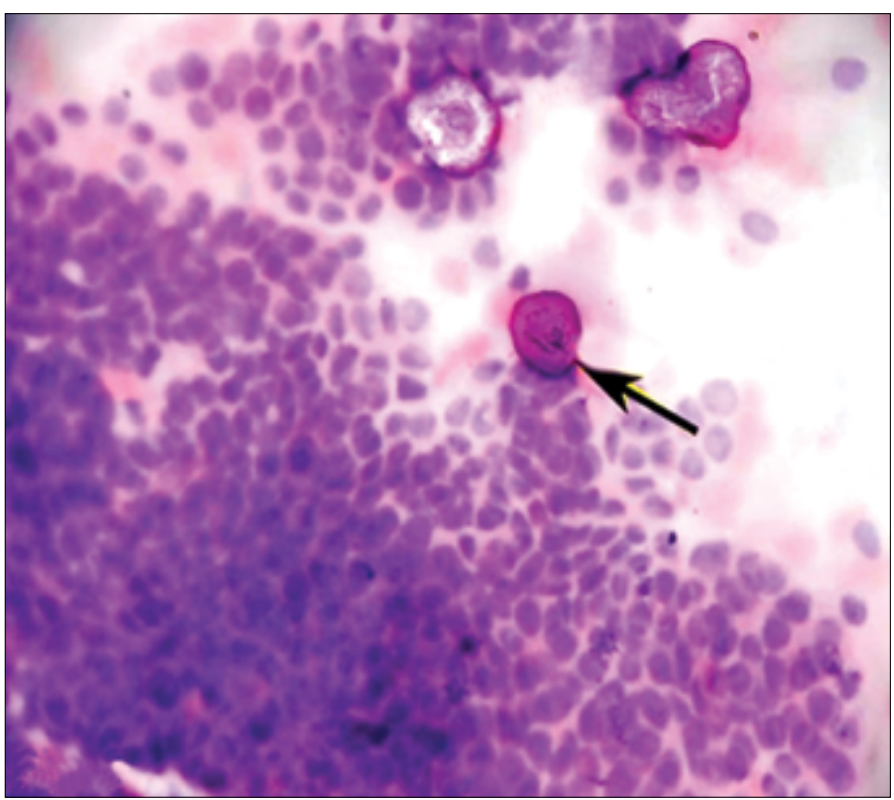

Figure 2. Papillary tissue fragments of malignant glandular cells, psammoma body (black arrow) (Papillary serous cystadenocarcinoma), H\&E, $40 \mathrm{X}$

mucinous neoplasms showed small clusters and isolated columnar epithelial cells with basally placed nuclei against a mucinous background. In most of the cases of serous and mucinous cystadenomas, specific diagnosis could be rendered on cytology. However, 2 cases of borderline mucinous tumour, 1 case of borderline serous tumour and 1 mucinous cystadenocarcinoma were diagnosed erroneously (Table 2). Papillary serous cystadenocarcinoma showed papillary fragments comprised of tumour cells with hyperchromatic nuclei and a high nucleo-cytoplasmic ratio. Psammoma bodies were identified in 2 cases (Figure 2). Mucin-producing cells with malignant nuclear features against a background of mucin was seen in mucinous cystadenocarcinomas (Figure 3a, b). One case of Brenner

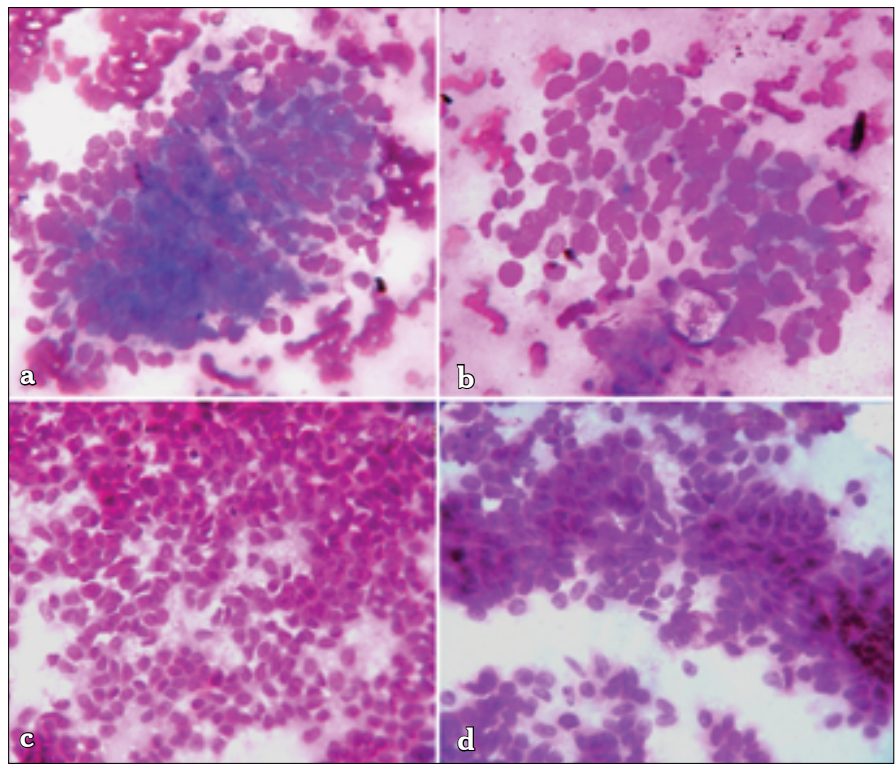

Figure 3. a-d. Mucinous cystadenocarcinoma. Mucin-producing cells in clusters, MGG, $40 \mathrm{X}$ (a). Mucinous cystadenocarcinoma. Epithelial cells with prominent nucleoli, MGG, 40 X (b). Granulosa cell tumour. Uniform round to ovoid cells with microfollicle formation, H\&E, $40 \mathrm{X}$ (c). Granulosa cell tumour. Loose cluster of cells with uniform, ovoid nuclei, some with grooves, H\&E, $40 \mathrm{X}$ (d)

tumour was misdiagnosed as mucinous cystadenocarcinoma on cytology (Table 1). Aspirate from a benign cystic teratoma showed mature squamous cells and degenerated cells in a dirty background, whereas fibroma showed a few tight clusters of benign plump spindle cells. The only case of granulosa cell tumour showed uniformly small round nuclei with microfollicle formation and nuclear grooves, which were more prominent in alcohol-fixed smears (Figure 3c, d, 4). Dispersed cells with distinct nucleoli and pale fragile cytoplasm, along with scattered lymphocytes in the background, were found in dysgerminoma. Yolk sac tumours, on the other hand, showed papillary clusters of cells with cytoplasmic vacuolation and pink globules. We also had one case of squamous cell carcinoma, possibly arising from a teratoma, which showed squamous cells with dense refractile cytoplasm and irregular hyperchromatic nuclei. Smears from 3 lesions that were cytologically labelled as 'suspicious of malignancy' showed monolayered sheets of epithelial cells with an increased nucleo-cytoplasmic ratio against a dirty background. However, mitotic figures and nucleoli were not conspicuous in these cases (Figure 4). Two cases belonging to this category were serous borderline tumours, while one case was a mucinous borderline tumour.

\section{Discussion}

Patients with ovarian masses, particularly those having malignant lesions, usually present with advanced disease. There are conflicting data regarding the diagnostic accuracy and safety of FNA (9-11). Aspiration cytology has been widely used method for the diagnosis of solid and cystic masses of the ovary. The 


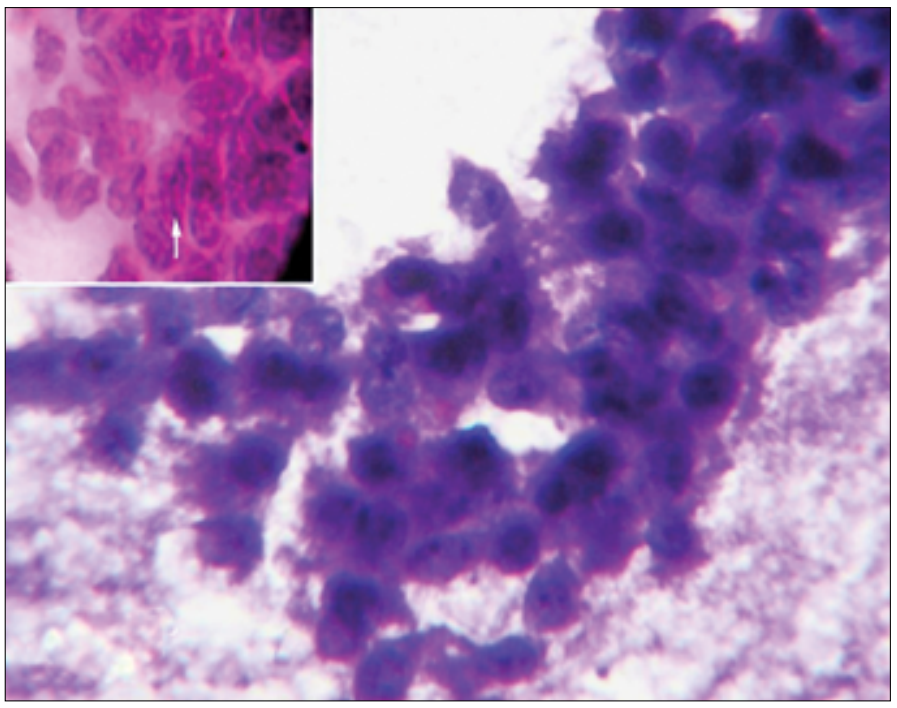

Figure 4. Monolayered sheets of epithelial cells with increased nucleo-cytoplasmic ratio and inconspicuous nucleoli suspicious of malignancy on cytology. Inset showing grooves (white arrow) in Granulosa cell tumour MGG, $40 \mathrm{X}$

procedure has been used for both the primary diagnosis of ovarian lesions and the follow-up of recurrent malignancies. Developing radiologic guidance techniques have also contributed to the higher accuracy of FNAC in recent years (2). This technique is used in other fields of medicine and of proven value in diseases of breast, thyroid and lung (12).

In gynaecologic oncology, FNAC has been used both for primary diagnosis and follow-up of recurrence in malignant ovarian lesions. The procedure is also accurate and safe for the diagnosis of disease that has metastasised to the lymph nodes, parametrium and vagina (13). Advancement of the radiologic guidance technique has contributed to the higher accuracy of FNAC in recent years (2). Gynaecologists are concerned about the safety of this procedure and the consequent upstaging of ovarian cancers. Zanetta et al. (14) reported fewer complications in a study of aspiration of 838 ovarian cysts. They concluded that FNAC can decrease the need for surgery in many women with ovarian cysts.

Owing to its complexity and the wide spectrum of diagnoses, cytological analysis of ovarian lesions is a difficult issue. However, differentiation into malignant and benign tumours is possible by the careful evaluation of the cytoarchitecture and background features (2). Pinpoint diagnosis can be made in a large number of cases. A critical issue of this procedure excluding its safety is regarding its accuracy. Information available in this regard projects some conflicting results. Ganjei \& Dickinson correctly diagnosed 9 out of 12 ovarian malignancies by FNAC; cytological examination correctly predicted all benign lesions of the ovary in their study, and they observed a sensitivity of $75 \%$ (15). Wojcik \& Selvaggi also reported that the majority of cystic ovarian lesions can be diagnosed accurately; however, they did not correlate FNAC with histology in 53\% of their cases (16). Aysun \& Canan compared the findings of FNAC and histology in ovar- ian masses and found a high sensitivity (95.1\%) and a specificity of $90.4 \%$ (2). Gupta and Rajwanshi found a sensitivity of $85.7 \%$ and a specificity of $98.0 \%$ (7). Cole and co-workers found FNAC to be highly specific (100\%) but conversely with a very low sensitivity of only $50 \%$ (8).

Our observations corroborate closely with those of other investigators, which indicates that FNAC can have appreciable sensitivity, specificity and accuracy in the diagnosis of ovarian masses. Higgins et al. (1) reported a specificity of $90 \%$ in the cytological evaluation of ovarian cysts, which is comparable to the present study, but showed a much lower sensitivity of $25 \%$. This may have been due to the inclusion of cystic ovarian lesions only in their study and the aspiration of cysts in postsurgical specimens.

The differences in the reported accuracy of cytological evaluation of ovarian masses may reflect the differences in the technique used to aspirate the lesion (transvaginal, transabdominal, laparoscopic or during laparotomy, with or without image guidance) as well as differences in smear preparation. Correlations with clinical parameters of the patients undergoing FNAC may be important, including serum markers and USG, as some studies included only women with a lower risk (11). USG can provide necessary clues towards the nature of the lesion: anechoic to hypoechoic lesions suggest a benign cyst, while solid cystic lesions with heterogeneous echogenicity indicate malignancy. Our study population included women with both cytological and histopathological materials available. Consequently, this study may be biased towards cases that are more suspicious clinically and patients requiring subsequent surgery.

Several other factors may explain a poor cyto-histopathological correlation. FNAC of an ovary may yield cyst fluid, ovarian cortex, ovarian stroma, or a combination of these structures. Ovarian cyst fluid may have occasional cells only (in a background of fluid) to provide an accurate impression of the lesion. Malignant cells in the ovary may not be uniformly distributed in the organ, and it can often be seen that cytological examination of the peritoneal washings in patients with known ovarian malignancy fail to identify malignant cells (1). Clinicians may have an unrealistic impression that interpreting ovarian cytological evidence is similar to analysing cytological findings from other organs. Ovaries have an incidence of an extensively diverse spectrum of primary tumours; hence, the impression on image guided cytology may not always accurately corroborate with the histopathology. In addition, borderline epithelial tumours may be difficult to interpret on aspiration cytology. Careful observation of a few small clusters of atypical cells with tumour diathesis may prompt a diagnosis towards 'epithelial lesion, suspicious of malignancy' in such cases. Many pathologists might not have the training and experience in the diagnosis of aspiration smears as they have in histopathological sections (1).

Fine-needle aspiration cytology provides some advantages for evaluating ovarian diseases, including excellent patient compliance and an extremely low complication rate. However, precisely categorising borderline tumours and false negative 
cytological analysis may be its limitations (2). Some studies indicate that the use of FNAC in ovarian cystic lesions can mislead the clinician about the nature of the cyst. If all types of ovarian lesions are analysed in sufficient numbers, it might improve the diagnostic accuracy (1).

Pelvic masses should be evaluated meticulously by laboratory, radiography and USG tests. Despite the lack of evidence, gynaecologists prefer exploratory laparotomy to FNAC due to the fear of peritoneal seeding from tumour cell spillage. FNAC of solid ovarian SOLs may play a useful role in determining tumour type and formulating management. Moreover, FNAC in patients with benign lesions like endometriosis or inflammatory masses may also lead to the patients being spared unnecessary surgery (15). Although the potential risk of seeding of an ovarian cancer during FNA has been mentioned in textbooks, only one reference was documented, 19 years ago (17-19). This was a series of 2 cases having a tumour which was believed to have spread due to the FNA performed during laparoscopy (20), while available recent literature remains silent in this regard.

Mulvany further reports in his study of 235 ovarian cyst aspirates than none of the 7 malignant cases in his series had an increased recurrence due to ovarian cyst aspiration (21). Hence, it seems reasonable to state that the potential threat of tumour spillage due to FNA has not been convincingly and adequately reported and the magnitude of such risk remains unknown (22). In addition, since more than two-thirds of malignant ovarian tumours present in an advanced stage, the concern for tumour dissemination seems much less important when weighed against the less invasive, effective and economical diagnostic tool available in the form of FNAC. In this clinical setting, neoadjuvant chemotherapy may be considered prior to cytoreductive surgery, and it is imperative that accurate diagnosis is required for such therapy. Although in this study the specificity of cytologic evaluation of ovarian cystic masses was comparable with other similar studies, a much higher sensitivity is desirable to identify women in whom subsequent surgical intervention may be unnecessary. FNA of ovarian cystic masses with $97 \%$ specificity and $83 \%$ sensitivity still surpasses other clinical parameters in the diagnosis of malignancy. Hence, the available clinical, radiological and laboratory findings may be combined with FNAC for an accurate preoperative diagnosis.

To conclude, image-guided FNAC is a quick, easy, fairly sensitive, specific and cost effective modality for the preoperative diagnosis of malignant as well as benign ovarian masses with minimal morbidity, pending histological confirmation. Dissemination and seeding of malignant cells during the procedure is not supported by adequate and conclusive literature. Targeted larger trials to address the issue of seeding of malignant cells during the procedure are needed to prove or disprove its debatable role in diagnosis. On the other hand, the procedure may help in avoiding unnecessary surgery or laparoscopy and making decisions regarding neoadjuvant chemotherapy; hence, it might be indispensible in this part of the world where most of the ovarian malignancies either present late in their course or as such no screening method is available. Accurately identifying borderline tumours and false negative cytological analysis due to low cellularity or secondary degenerative changes may be its limitations.

Ethics Committee Approval: Ethics committee approval was received for this study.

Informed Consent: Written informed consent was obtained from patients who participated in this study.

Peer-review: Externally peer-reviewed.

Author contributions: Concept - S.R., M.G.; Design - S.R., M.G., K.M.; Supervision - N.C., S.R.; Resource - A.B., S.R., M.G.; Materials - A.B., M.G.; Data Collection\&/or Processing - A.B., S.R.; Analysis\&/or Interpretation - M.G., K.M., A.B.; Literature Search - M.G., K.M., S.R.; Writing - S.R., M.G., K.M.; Critical Reviews - N.C., M.G.

Conflict of Interest: No conflict of interest was declared by the authors

Financial Disclosure: The authors declared that this study has received no financial support.

\section{References}

1. Higgins RV, Matkins JF, Marroum MC. Comparison of fine-needle aspiration cytologic findings of ovarian cysts with ovarian histologic findings. Am J Obstet Gynecol 1999; 180: 550-3. [CrossRef]

2. Uguz A, Ersoz C, Bolat F, Gokdemir A, Vardar MA. Fine needle aspiration cytology of ovarian lesions. Acta Cytol 2005; 49: 144-8. [CrossRef]

3. Kreuzer GF, Paradowski T, Wurche KD, Flenker H. Neoplastic or Nonneoplastic ovarian cyst? The role of cytology. Acta Cytol 1995; 39: 882-6.

4. Sevelda P. Prognostic influence of intraoperative rupture of malignant ovarian tumors. Presented at the first European Congress of Gynecologic Endoscopy, France: Clermont-Ferrand; September 9-11, 1992.

5. Geier GR, Strecker JR. Aspiration Cytology and E2 content in ovarian tumors. Acta Cytol 1981; 25: 400-6.

6. Mehdi G, Maheshwari V, Afzal S, Ansari HA, Ansari M. Image-guided fine-needle aspiration cytology of ovarian tumors: an assessment of diagnostic efficacy. J Cytol 2010; 27: 91-5. [CrossRef]

7. Gupta N, Rajwanshi A, Dhaliwal LK, Khandelwal N, Dey P, Srinivasan R, Nijhawan R. Fine needle aspiration cytology in ovarian lesions: an institutional experience of 584 cases. Cytopathology 2012; 23: 300-7. [CrossRef]

8. Lisa Cole, Sharon Mount, Erica Nuzzo, Cheung Wong . Aspiration cytology of ovarian cystic masses: histologic correlation and review of the literature. Acta Cytol 2011; 55: 19-25. [CrossRef]

9. Moran O, Menczer J, Ben-Baruch G, Lipitz S, Goor E. Cytologic examination of ovarian cyst fluid for the distinction between benign and malignant tumors. Obstet Gynecol 1993; 82: 444-6.

10. Dordoni D, Zaglio S, Zucca S, Favalli G. The role of sonographically guided aspiration in the clinical management of ovarian cysts. $\mathrm{J}$ Ultrasound Med 1993; 12: 27-31.

11. Papathanasiou K, Giannoulis C, Dovas D, Tolikas A, Tantanasis T, Tzafettas JM. Fine needle aspiration cytology of the ovary: is it reliable? Clin Exp Obstet Gynecol 2004; 31: 191-3. 
12. Hajdu S, Melamed M. Limitations of aspiration cytology in the diagnosis of primary Neoplasms. Acta Cytol 1984; 28: 337-45.

13. Belinson JL, Lynn JM, Papillo JL, Lee K, Korson R. Fine needle aspiration cytology in the management of gynecologic cancer. Am J Obstet Gynecol 1981; 139: 148-53.

14. Zanetta G, Trio D, Lissoni A, Dalla Valle C, Rangoni G, Pittelli M, et al. Early and short term complications after US-guided puncture of gynecologic lesions: evaluation after 1000 consecutive cases. Radiology 1993; 189: 161-4.

15. Ganjei P, Dickinson B, Harrison T, Nassiri M, Lu Y. Aspiration cytology of neoplastic and non-neoplasic ovarian cysts: Is it accurate? Int J Gynecol Pathol 1996; 15: 94-101. [CrossRef]

16. Wojcik EM, Selvaggi SM. Fine needle aspiration cytology of cystic ovarian lesions. Diagn Cytopathol 1994; 11: 9-14. [CrossRef]
17. Bibbo M, Wood MD, Fitzpatrick BT. Peritoneal Washings and Ovary. In: Bibbo M, Wilbur D, editors. Comprehensive Cytopathology. Amsterdam, Holland: Saunders Elsevier; 2008.p.294. [CrossRef]

18. Cibas ES. Ovary. In: Cibas ES, Ducatman BS, editors. Cytology Diagnostic Principles and Clinical Correlates. New York, USA: Saunders; 2003.p.406.

19. Koss LG. Tumors of the Ovary and Fallopian Tube. In: Koss LG, Melamed, editors. Koss' Diagnostic Cytology and its Histopathologic Bases. New York, USA: Lippincott Williams \& Wilkins; 2006.p.493.

20. Trimbos JB, Hacker NF. The case against aspirating ovarian cysts. Cancer 1993; 72: 828-31. [CrossRef]

21. Mulvany NJ. Aspiration cytology of ovarian cysts and cystic neoplasms: a study of 235 aspirates. Acta Cytol 1996; 40: 911-20. [CrossRef]

22. Ganjei P. Fine-needle aspiration cytology of the ovary. Clin Lab Med 1995; 15: 705-26. 